\title{
Henry Bauchau : de la nécessité dans la création
}

Il y a quelque temps, alors que je venais d'achever L'Enfant bleu ${ }^{\mathbf{1 3 0}}$, j'ai entrepris la lecture d'un autre ouvrage ${ }^{131}$, sans aucun rapport apparent, consacré à la physique quantique, qui est peut-être l'aspect le plus mystique et/ou le plus ésotérique de la science actuelle. On y expliquait, pour simplifier, que la rencontre des constituants d'un atome, les particules élémentaires, crée, non pas du même, mais quelque chose de complètement différent, d'abord virtuel car non observable, puis bien réel, bien concret, bien vivant. Et vraiment tout autre. Quel rapport avec la création envisagée par Henry Bauchau? Le lien entre cette lecture et L'Enfant bleu m'est apparu de façon flagrante: ce récit si émouvant est celui d'une rencontre, celle d'un enfant et d'une adulte, qui permet l'émergence, l'éclatement d'une intense et dense création artistique, insoupçonnée. C'est aussi la rencontre entre l'énergie vibratoire des ouvres ainsi créées et celle des êtres qui les recevront, contempleront, écouteront, toucheront dans l'espace et le temps du monde et celui de l'esprit, de l'âme et du cœur.

Véronique, psychothérapeute, prend en charge Orion, un adolescent psychotique. L'Enfant bleu est l'histoire d'une naissance à soi: Orion peu à peu s'approprie un "je" grâce à la présence de tous les instants de sa thérapeute, qui suscite, dans d'intenses séances de thérapie, une création verbale et graphique d'une grande richesse. Mais le récit de L'Enfant bleu n'est pas seulement cela. Outre les moments de transfert et contre-transfert évoqués dans ces pages, c'est le cheminement de l'entourage proche d'Orion qu'Henry Bauchau éclaire. Lacte thérapeutique tel qu'évoqué par l'écrivain m’a fait songer à un lancer de caillou dans l'eau calme d'une rivière. Après l'impact, on observe se former en silence de larges ronds en un mouvement centrifuge qui viennent bousculer la libellule qui reposait pourtant bien plus loin sur la végétation aquatique. Ainsi en est-il de L'Enfant bleu où les cheminements intérieurs de l'enfant et de l'adulte se touchent et fonctionnent même, nous le verrons plus loin, en miroir. D'ailleurs, dans de nombreux passages du récit, on glisse souvent et subrepticement de l'enfant à l'adulte dans un même développement, un même paragraphe.

130 Henry Bauchau, L'Enfant bleu, Arles, Actes Sud, 2004.

131 Sven Ortoli et Jean-Pierre Phrarabod, Le Cantique des quantiques: le monde existe-t-il?, Paris, La Découverte, 2007. 
Mais arrêtons-nous un instant sur le prénom du jeune garçon: "Orion". Outre la référence au chasseur de la mythologique grecque, en astronomie, Orion est une étoile qui s'insère dans un ensemble qui forme une figure, qu'on appelle constellation, facilement observable dans le ciel clair hivernal de par la présence de quelques étoiles de forte magnitude. Au sein de cette constellation se trouve la nébuleuse du même nom. Comme toutes les nébuleuses, elle a la particularité d'être une pouponnière d'étoiles en formation, en devenir. Par la densité de l'énergie et de la matière ainsi réunies, par l'interaction des unes avec les autres, les étoiles vont naître et mourir, car cette nébuleuse contient aussi des étoiles en fin de vie. Ainsi Orion regroupe-t-elle toutes les étapes de la vie. Elle renferme donc à elle seule tout le mystère de la Création.

Sachant cela, si l'on accepte avec Hermès Trismégiste ${ }^{\mathbf{1 3 2}}$, que ce qui est en haut est comme ce qui est en bas, on comprend d'autant mieux que L'Enfant bleu soit le récit d'une croissance intérieure où la rencontre avec l'Autre et toutes les difficultés qu'elle comporte fait naître à soi tout en permettant, dans un second temps, l'émergence d'autre chose que soi, d'autre chose que le "moi". Il s'agit d'une sorte d'effacement de soi, pour rendre grâce à ce qui ne peut être nommé, "quelque chose" qui est donné ou rendu à la communauté humaine. C'est ce que je propose de montrer dans mon intervention: sa rencontre avec l'Autre permet à Orion de créer, permet le prodige de la création artistique, un Univers jusqu'alors inconnu de soi-même et tout autre, un monde animé de sa propre vie et, j'insiste ici, qui poursuit sa vie indépendamment de celui qui l'a engendré, car ce qui est offert alors est la possibilité pour chacun de se voir dans, de parler au travers de, et sans doute aussi de s'effacer avec si l'on accepte de considérer avec moi qu'Henry Bauchau, dans le sillage de Simone Weil, souscrit à l'idée selon laquelle "l'œuvre d'art, quand elle est parfaite, a quelque chose d'essentiellement anonyme. Elle imite l'anonymat de l'art divin "133.

Mais revenons un instant au défi auquel est confronté Orion, dans un premier niveau de lecture. Il s'agit de parler à partir d'un "je", d'habiter pleinement un "moi", afin de trouver une place juste dans le monde, afin d'avoir un endroit à partir duquel exprimer ce qu'il est. La question ne semble pas se poser lorsqu'on est dit bien-portant, sain. Pourtant, cette question essentielle, Henry Bauchau l'aborde dans l'ensemble de son œuvre, qu'elle soit peinte ou écrite. Il l'envisage à travers tous les personnages qu'il crée. Dans L'Enfant bleu, ce défi est la justification essentielle de l'art-thérapie. Toute démarche psychothérapeutique est une voie exigeante pour celui qui s'y

132 Louis Ménard, Hermès Trismégiste: traduction complète précédée d'une étude sur l'origine des livres hermétiques, Paris, Guy Trédaniel éditions, 1990.

133 Simone Weil, La Pesanteur et la grâce, Paris, Plon, 1998, p. 169. 
risque. Puiser à l'endroit "juste", puiser à l'endroit de l'Inconscient que le Conscient ne sait comment vivre, crée un étourdissement, voire un affaiblissement des fonctions vitales; c'est un coup de boutoir pour le corps, car l'énergie ainsi déchargée est d'une telle puissance qu'avant l'émergence d'un nouvel équilibre, tout en soi est dans la précarité, le déséquilibre. Une thérapie, c'est prendre le risque d'aller mieux. Cela peut sembler paradoxal. Comment hésiter à aller mieux? Mieux que personne, Henry Bauchau, patient puis thérapeute, sait que se défaire de ses schémas de pensée habituels, routiniers presque, c'est prendre le risque d'avancer sans filet, c'est perdre ses repères, c'est le vertige du vide. L'abandon des résistances est le plus grand défi d'une démarche thérapeutique. L'Enfant bleu met cette résistance en lumière de façon tout à fait étonnante, car elle est très difficile à dire.

Or, il apparaît qu'à l'émergence d'un "Je" en Orion correspond un foisonnement créatif, l'expression d'une vie vibrante, d'une authenticité dont le corps est le premier réceptacle. Il faut "en passer par" le corps, l'habiter. C'est la réception du monde par le corps qui distingue le vivant du mort. Henry Bauchau montre ainsi, dans L'Enfant bleu, qu'est vivante toute ouvre d'art qui ouvre en l'homme un champ de perception corporelle, un espace vibratoire particulier. Et pour éclairer cela, nous évoquerons, trop succinctement hélas, un point de convergence assez remarquable entre ce que j'ai perçu de la réflexion d'Henry Bauchau dans L'Enfant bleu et l'approche d'Antonin Artaud, notamment pour ce qui a trait à l'expression musicale, qu'il vaudrait la peine de développer plus longuement dans des travaux ultérieurs.

Les deux approches ont ceci de commun que dans leur conception la puissance et la "justesse" de l'expression artistique se mesurent à leur réception par et dans le corps de celui qui crée, et de celui qui reçoit. La qualité particulière d'une création artistique accomplie mise au monde, donnée au monde, se mesure à sa réception corporelle par l'auditoire ou le spectateur. Si le corps ne vacille pas sous l'énergie vitale décuplée de la création, c'est le signe d'un manque, d'une non-incarnation de l'œuvre. C'est le signe d'une œuvre inerte, une œuvre mort-née.

Il suffit de prendre l'exemple de la création musicale, omniprésente dans L'Enfant bleu. La véritable passion de Vasco, l'époux de Véronique, est la musique. Pourtant, celle-ci remarque avec dépit:

[L]a musique [de Vasco] est parfaite mais est-ce vraiment la sienne?

C'est beau et pourtant nos corps ne sont pas émus ${ }^{134}$.

134 Henry Bauchau, L'Enfant bleu, op. cit., p. 188. 
Puis, Gamma, violoniste de talent et patiente d'un confrère de Véronique, décide d'adapter la musique de Vasco au violon:

Gamma commence, c'est le même morceau, ce n'est pas la même musique, ce sont les mêmes notes mais chargées d'une autre intensité. Gamma ne s'adresse pas au goût, à l'intelligence, à notre culture musicale. Elle parle à nos corps, un langage plus chaud, plus brûlant, au bord de la souffrance. Je suis à côté d'elle. Je vois qu'elle saute des notes, des accords, qu'elle crée des béances, des discordances qui font vivre plus fort la musique. Ce n'est plus la beauté qui est cherchée, ni trouvée, plus l'harmonie, c'est autre chose. Qui n'est pas atteint, qui ne peut pas l'être dans le bonheur mais qui existe par la musique dans la présence et l'au-delà du malheur. ${ }^{135}$

Ainsi, dans ce passage, l'improvisation de Gamma ouvre un champ de perception qui vient habiter le corps et le faire vaciller. Étonnant chavirage des corps à travers les vibrations des cordes. Au sens propre comme au sens figuré, la musique plonge l'auditoire dans une nouvelle perception et un nouvel état vibratoire, produits par des ruptures mélodiques, des écarts de tons, des "béances" et "discordances" qui font singulièrement songer aux recommandations d'Artaud dans son Théâtre de la Cruauté.

À de nombreuses reprises, ce dernier s'est penché sur les spécificités de l'art musical. Certes, il n'est pas le seul à s'être livré à cette réflexion. De nombreux compositeurs et penseurs du XIX siècle $^{136}$ et des premières années du XX ${ }^{\mathrm{e}}$ siècle l'ont fait avant lui. Mais la nature même de son théâtre exigeait "d'agir directement et profondément sur la sensibilité par les organes et cela, poursuit Artaud, invite, du point de vue sonore, à rechercher des qualités et des vibrations de sons absolument inaccoutumées [... „137. Artaud souhaitait mêler aux représentations des sons, des bruits grinçants qui s'élèveraient du spectacle, parce que, selon lui, "on peut par formes, par sons, musiques et volumes, évoquer [...] des sortes d'états d'une acuité [...] intense, d'un tranchant [...] absolu "138. Artaud évoque encore des intensités de sons qui "utilisent la vibration, la trépidation, la répétition [...] d’un rythme musical [qui] ne peuvent obtenir leur plein effet que par l'utilisation des dissonances" ${ }^{139}$.

De par son intensité même, de par son "trop plein", cette perception crée de la douleur. La douleur nourrit cette perception qui ne s'y installe pas, la dépassant même. La musique, créée et perçue de la sorte est l'expression

$135 \mathrm{Idem}$

136 On pense ici à Schopenhauer, Nietzsche ou Wagner parmi d'autres, qu'Artaud a lus avec grand intérêt.

137 Antonin Artaud, Euvres complètes, vol. IV, Paris, Gallimard, 1978; 1 1e éd. 1964, p. 92.

138 Ibid., p. 61

$139 \mathrm{Ibid}$., p. 121. En italiques dans le texte. 
d'un Instant à la fois pleinement ancré dans le Présent et hors du temps, une brèche ouverte sur un Ailleurs soudain à sa portée, donc toujours LÀ, qui attend, qui l'attend. Qui NOUS attend.

Dans L'Enfant bleu, comme dans de nombreux romans et nouvelles bauchaliens, lorsque l'artiste s'exprime d'une part de vérité en soi, il soigne l'Autre et se soigne lui-même malgré l'exigence physique d'un tel don. Si nous revenons au personnage de Gamma dans le roman, ce qui émerge de sa musique est la pleine expression de ce qui EST en elle, au-delà des filtres du Conscient et vient toucher l'Autre de la même façon. L'expression du potentiel créatif, la rencontre avec cette force est d'une violence telle que le corps physique risque l'épuisement, lorsque le don est authentique et non filtré. Le lâcher prise nécessaire, l'abandon des peurs et de la Raison ne sont pas sans risque. Gamma en avertit Véronique, alors que sur un poème de celle-ci, les deux musiciens ont improvisé, Gamma avec sa voix, Vasco au saxophone:

[...] Hier [Vasco] a montré à tous le grand musicien qu'il est.

- Grâce à toi [répond Véronique].

- Grâce à ton poème. Grâce à nous deux. Je peux l'aider à se réaliser [...] mais ce sera dangereux pour toi. [...] Méfie-toi, moi aussi je suis dangereuse, je mène ma vie vers le danger, comme il faisait avant toi, comme il devra le faire à nouveau. ${ }^{140}$

De fait, on se souvient d'un moment, dans le roman, où Gamma tombe malade lors d'un concert à l'étranger. La racine de ce mal n'est pas clairement identifiée dans le récit et le lecteur se perd en conjectures: l'épuisement sans doute, tant la démonstration de la jeune femme, lors de l'improvisation au violon, a exigé d'elle une énergie particulière. On comprend d'autant mieux qu'on puisse alors souhaiter résister. On peut vouloir refuser cette exigence en usant de toutes sortes de stratégies d'évitement. Avant d'obéir à ce qui sourd en lui, Vasco, l'ingénieur en mécanique automobile, résiste violemment à la dureté du combat qui exige la défaite de la logique, des connaissances acquises, des habitudes et des schémas de pensée:

De nouveau les rêves, les sommets, les gouffres, les naufrages, l'immense patrie des illusions. Notre pauvre existence, notre art éphémère à la cime des montagnes et le vent qui fait là-dedans sa musique géante. C'est trop, Véronique, cette conception épique, hérö̈que de la vie et de la musique, c'est trop pour moi. Vive les moteurs, leur précision, leur force maitrisée par le calcul et l'expérience. Ta conception de la musique, de l'art, ce n'est plus la vie, c'est de l'épopée. Finie l'épopée ${ }^{141}$.

140 Henry Bauchau, L'Enfant bleu, op. cit., pp. 193-194.

141 Ibid., p. 212. 
Cette résistance à l'Inconnu, cette façon de se cramponner au connu qui sécurise même s'il est inconfortable lui aussi, c'est toute l'expérience douloureuse et la difficulté du travail thérapeutique, dont Henry Bauchau rend parfaitement compte dans son récit.

Dans l'œuvre peint et dans les écrits d'Henry Bauchau, l'Art rend précisément compte d'un combat, des "résistances" de l'artiste ${ }^{142}$, d'un trésor, au départ "enseveli " ${ }^{143}$, qui lentement prend corps, et du "génie sauvage " ${ }^{144}$ qui habite tout artiste. C'est ce parcours qui est évoqué dans L'Enfant bleu, à travers Orion, si touchant, mais aussi à travers Vasco, dont les résistances fonctionnent en miroir de celles d'Orion. Ainsi, est "malade" tout être qui fuit ce qu'il est vraiment. Véronique l'exprime en termes dépourvus d'ambiguité dans un passage où Vasco lutte contre ce qui sourd en lui:

La musique c'est toi. Toi! C'est ta vie que tu fuis. Tu n'en as pas d'autre, tu crois avoir le choix, tu ne l'as pas, le choix est fait. Tu feras la musique des profondeurs, celle qu'on ne commande pas, qu'on ne maîtrise pas, ou rien. [...]. C'est ainsi, c'est tout. ${ }^{145}$

C'est une impérieuse nécessité au sens où l'entend Simone Weil. C'est une nécessité difficile mais imparable, et qui résume assez bien, à mon sens, la façon dont Henry Bauchau envisage la vie humaine. Simone Weil a formulé cela tout aussi clairement dans La Pesanteur et la grâce, rendant la parenté philosophique et spirituelle sinon évidente, tout au moins, assez intéressante entre eux. Elle écrit: "Au moment où l'on prend conscience qu'il y a un choix à faire, le choix est déjà fait - dans un sens ou dans l'autre". Voilà qui est imparable. Henry Bauchau, me semble-t-il, ajoute à cela que le défi de toute création artistique et, de façon plus générale, de toute existence, est de devenir soi-même, quel qu'en soit le tribut, quelle qu'en soit la violence ou le déséquilibre. Seulement ensuite s'agit-il de s'effacer ou de se fondre, de disparaître dans l'œuvre et de la donner ainsi en partage à tous, à chacun, au Tout.

En effet, Henry Bauchau rend bien compte, comme la philosophe et mystique, qu'il n'y a pas d'autre voie possible, pas d'autre choix que de se soumettre à une forme d'obéissance. Simone Weil évoque l'obéissance soit à «la pesanteur", soit "au rapport des choses" "146. "Dans le premier cas, poursuit la philosophe, on fait "ce à quoi pousse l'imagination combleuse de vides. On peut y mettre [...] toutes les étiquettes, y compris le bien et Dieu "147. Dans

142 Ibid., p. 192.

143 Idem.

144 Idem

145 Ibid., p. 212.

146 Simone Weil, op. cit., p. 60

147 Idem 
le second cas, "on fixe l'attention sur le rapport des choses", une nécessité apparaît alors à laquelle "on ne peut pas ne pas obéir" ${ }^{148}$. Orion, Vasco, tout artiste, et plus largement tout homme est face à ce choix. Henry Bauchau montre les artistes de L'Enfant bleu "poussés par" une force nécessaire. En ce sens, les dessins d'Orion ne sont pas l'expression de l'imagination au sens weilien du terme, dans le sens où l'enfant DOIT écrire et dessiner. Il n'en a pas le désir. "L'imagination est toujours liée à un désir, c'est-à-dire à une valeur. Seul le désir sans objet est vide d'imagination "149, écrit Simone Weil.

Détachement des fruits de l'action [...] Agir, non pour un objet, mais par une nécessité [...]. Transporter hors de soi les mobiles de ses actions. Être poussé [...] Pour tout acte, le considérer sous l'aspect non de l'objet, mais de l'impulsion. Non pas : à quelle fin? Mais d'où cela vient-il ?150

L'artiste, tel que l'envisage Henry Bauchau est celui qui obéit à la nécessité. Il ne fait pas pour, mais est poussé par. Dans son extrême innocence, Orion crée, plongé dans ce type d'élan, d'énergie. Il va puiser dans la source intérieure, sans finalité particulière, bien que Véronique, elle, cherche à lui faire revêtir un "moi", un "je", parce qu'il faut en passer par là pour accéder, ensuite, à ce qui n'est plus ce "je». Aussi le mouvement est-il inverse pour Orion et Vasco: Orion doit "habiter", trouver l'endroit d'où dessiner et écrire et Vasco doit le perdre, afin d'accéder à ce qui est au-delà ou ce qui est au-dedans de la création, donnée ensuite en partage à l'anonyme regard de l'Autre. En somme, Orion et Vasco fonctionnent en miroir d'une certaine façon, le but étant cependant le même: il faut prétendre au "moi", à un moi authentique, avant sa dissolution, avant d'être Vivant dans l'effacement, si on veut bien me suivre sur ce terrain.

Si Henry Bauchau montre selon moi, qu'il faut "en passer par là ", qu'il faut habiter le "je" avant de consentir à le perdre, rien mieux que Le Boulevard périphérique ${ }^{151}$ ne l'explicite. En effet, le récit montre ce qu'il advient de celui dont le moi a été détruit. Je fais allusion bien évidemment à la figure dense de Shadow dans son absolue noirceur. Il s'agit d'un homme dont le "je" est mort, dont le "je" a été tué, massacré par l'histoire familiale. Et une fois encore, le recours à la pensée de Simone Weil, qui, à mon sens, irrigue les pages du roman, éclaire mes propos et jette une lumière intéressante sur Shadow:

148 Idem.

149 Ibid., p. 78.

150 Ibid., p. 56.

151 Henry Bauchau, Le Boulevard périphérique, Arles, Actes Sud, 2008. 
Pour ceux dont le je est mort, on ne peut rien faire, absolument rien. Mais on ne sait jamais si, chez un être humain déterminé, le je est tout à fait mort ou seulement inanimé. S'il n'est pas tout à fait mort, l'amour peut le ranimer comme par une piqûre, mais seulement l'amour tout à fait pur, sans la moindre trace de condescendance, car la moindre nuance de mépris précipite vers la mort. Quand le je est blessé du dehors, il a d'abord la révolte la plus extrême, la plus amère, comme un animal qui se débat. Mais dès que le je est à moitié mort, il désire être achevé et se laisser aller à l'évanouissement. Si alors une touche d'amour le réveille, c'est une douleur extrême et qui produit la colère et parfois la haine contre celui qui a provoqué cette douleur. De là, chez les êtres déchus, ces réactions en apparence inexplicables de vengeance contre le bienfaiteur. ${ }^{152}$

Le "je" moribond de Shadow, face à la grâce de Stéphane, ne peut que détruire l'insupportable amour qui est venu lui rappeler son état de ruine intérieure.

Henry Bauchau, dans sa grande humanité, son regard empli de compassion, montre récit après récit, des visages d'hommes, de femmes, d'enfants soumis à la pesanteur, qui tâchent de ne pas courber le dos, mus par la nécessité, qui les pousse à devenir ce qu'ils sont, à obéir. Parmi ces figures emblématiques, se dresse la frêle silhouette d'Antigone bien sûr. Une autre figure emblématique de l'obéissance est, à mon sens, l'artiste, car il accomplit la descente vertigineuse dans les profondeurs de l'être pour toucher au cœur de celui-ci, et où, par là-même, l'identité s'efface, afin de rapporter de cette plongée dans l'abysse ce qui est indicible, ce qui n'a pas de nom et qui n'est plus le "moi".

Il me semble que dans la conception bauchalienne, celui qui crée ne cherche pas à "boucher toutes les fissures par où passerait la grâce "153, pour reprendre les mots de Simone Weil. Il est en effet dans l'Acceptation de sa condition d'être humain incarné mais allégé de par la connaissance qu'il a d'être aussi lourd. Il accepte l'enracinement sans le vivre comme une défaite. Il accepte, dans une forme de simplicité et aussi de noblesse extrême, d'être sur la Terre, mais jamais vraiment loin du Ciel. Il est dans l'acceptation de la vie dans ses déchirantes contradictions. "Comment supporter cette vie partagée entre le doute et l'espérance, comment ne pas la

152 Simone Weil, op. cit., p. 38. Alors que s'établit, dans les œuvres de jeunesse une distinction entre le "moi" et le "je, les différences entre les deux semblent s'abolir au fil des textes des dernières années. A lire sur ce point, l'excellente et riche introduction de Florence de Lussy aux Euvres de Simone Weil (Paris, Gallimard, 1999, pp. 11-33).

153 Ibid., p. 25 
supporter?" s'interroge le narrateur du Boulevard périphérique ${ }^{154}$. Avant lui, Simone Weil a fait de la condition finie de l'humain qui aspire à l'infini la plus déchirante des contradictions, la menant ainsi à interroger les racines du Mal. Antonin Artaud, plus violemment, s'est insurgé lui aussi contre ce qui lui semblait un scandale insurmontable. On pourrait évidemment citer bien d'autres noms, mais j'ai choisi ces deux illustres figures de par leur évidente filiation avec Henry Bauchau.

\section{La figure de l'arbre}

Pour rendre compte de cet écartèlement, du double mouvement vertical et horizontal, d'enracinement/déracinement ${ }^{155}$, pour rendre compte de ce qui grandit en soi, ou/et de ce qui se coupe ou s'élague en soi à coups de hache, avec violence, pour s'exprimer dans l'Art, Henry Bauchau fait appel à la figure de l'arbre.

Au-delà de l'image de la croix et de la souffrance christique, ou même de l'idée manichéenne ${ }^{156}$ selon laquelle le corps spirituel et l'âme du Premier homme ont été dispersés à travers la matière, crucifiés sur l'étendue de la matière, l'arbre explicite ce qui est à l'œuvre dans l'exploration de la psyché. Larbre, c'est l'Homme, qui plonge ses racines dans l'humus de la mémoire du passé et de l'Inconscient, tout ce qui est souterrain, obscur, là où il fait noir, là où on a peur. Mais l'arbre c'est aussi l'Homme qui s'élève vers la lumière, qui puise dans le gouffre l'élan, l'irrésistible ascension vers... Mouvement ascendant et descendant: "La création est faite du mouvement descendant de la pesanteur, du mouvement ascendant de la grâce et du mouvement descendant de la grâce à la deuxième puissance "157 écrit Simone Weil.

Dans L'Enfant bleu, comme dans de nombreux autres récits, l'arbre est omniprésent. Dans le roman, il est présent dans tous les épisodes qui marquent les paliers de croissance de Véronique, d'Orion ou de Vasco, cercles concentriques marqués dans la chaire de l'arbre, sous l'écorce. Autres cercles centrifuges dans leur rayonnement secret.

Ainsi, associé à l'écriture poétique de Véronique, le morceau musical qui révèle Vasco et dont il est le compositeur s'intitule "Larbre d'Homère "158:

154 Henry Bauchau, Le Boulevard périphérique, op. cit, p. 67.

155 Simone Weil évoque l'enracinement "dans l'absence de lieu", dans La Pesanteur et la grâce, op. cit., p. 50 .

156 À lire la passionnante étude de François Decret, Mani et la tradition manichéenne, Paris, Seuil, 2005.

157 Simone Weil, op. cit. p. 10.

158 C'est aussi le titre d'un poème d'Henry Bauchau paru en 1982 dans Heureux les déliants (Bruxelles, Labor, 1995). 
Est-ce que Vasco est délivré, est-ce qu'il a libéré sa musique? Est-ce qu'il ne se laissera plus couper d'elle par [...] les ordres impérieux du savoir? Sous la voix de Gamma, j'entends celle de Vasco qui crie dans son saxo: non je n'abandonnerai plus l'arbre d'Homère. Oui, promet la voix insurgée de Gamma, les aveugles, les navrés, les psychotiques peuvent chanter et partager avec tous leur amour. Homère chante à deux voix, celle de Vasco engendre le dieu des combats et de la dure nécessité. Celle de Gamma, espère et aime: l'arbre qu'on croyait mort, il est vivant, peut-être... ${ }^{159}$

Certes on reconnaît ici toute la source vive que représentent la littérature et la civilisation de la Grèce ancienne pour Henry Bauchau. Et on aborde là un autre aspect intéressant de l'œuvre de l'écrivain: l'indissociable alliance entre une forme de cécité et la pénétrante vision de ce qui EST par le toucher, par le corps.

La cécité est une valeur presque intrinsèque à l'artiste vrai. Il s'agit de Voir autrement, non plus avec la perception visuelle et tout son champ de lecture et de représentations, mais de toucher. On voit en touchant. Il y aurait beaucoup à dire sur la cécité comme manière de Voir, de créer ou de voir en l'Autre, et pas seulement si l'on est artiste. Outre CEdipe, personnage central de nombreuses nouvelles de Bauchau, la cécité apparaît en filigrane dans d'autres nouvelles, dont En Noir et blanc ${ }^{160}$, dans laquelle la femme avec qui s'entretient le narrateur est "à demi aveugle". Elle a su "voir" l'artiste en sommeil dans l'homme avec lequel elle s'entretient: "je pressentais autre chose en vous, peut-être l'œuvre que vous avez faite. Qui s'est faite à travers moi. Si vous voulez. Mais vous l'avez laissée faire. Je sentais ce noyau dur en vous qui voulait irréductiblement grandir " ${ }^{\mathbf{1 6 1}}$. On retrouve ici l'idée maîtresse selon laquelle l'œuvre se fait à travers l'artiste qui a agi selon une instance intérieure impérieuse et nécessaire.

Dans L'Enfant bleu, cette nécessité s'exprime par la pratique du dessin mais aussi par celle de la sculpture, ce qui semble tout à fait cohérent avec la cécité comme façon de voir autrement. En effet, puisque l'on voit en touchant, cet art du vivant rassemble toute la symbolique et toute la lutte qui précède l'incarnation matérielle, concrète, de ce qui, en soi, demeurait virtuel, non observable, non exprimé et qui, une fois l'œuvre faite, poursuit sa vie secrète dans le corps et les yeux de celui qui touche et regarde, qui suit les courbes, les contours du matériau sculpté.

159 Henry Bauchau, L'Enfant bleu, op. cit., p. 191.

160 Henry Bauchau, En Noir et blanc, Paris, Ed. du Chemin de fer, 2005.

161 Ibid., p. 40. 
Dans l'une des nouvelles qui composent Les Vallées du bonheur profond, intitulée "Larbre fou", CEdipe et Antigone ont trouvé refuge chez Diotime pour l'hiver. Poussé par Clios, Edipe entreprend de faire une statue d'un arbre foudroyé par l'orage. Avant de sculpter l'arbre choisi, en une gestuelle presque rituelle, CEdipe enlace l'arbre qu'il s'apprête à sculpter: "CEdipe s'approche du tronc, le palpe longuement, le touche et l'enserre de tout son corps" "162. Tout se passe comme si de la force vivante de l'arbre, "quelque chose " attendait d'être révélé et extirpé. Lorsqu'Antigone demande à CEdipe ce qu'il va créer, celui-ci "touche [l'arbre] avec une sorte d'effroi: "Ce qui est là, la danse sauvage. [...] Je le ferai à la hache” "163. Mais c'est bien évidemment tout autant quelque chose de soi qu'il s'agit d'extirper. Les paroles d'Antigone éclairent le travail à l'œuvre: "[Diotime] dit qu'il faut que tu réfléchisses et sondes ta colère entre chaque coup "164.

On assiste ensuite à la création elle-même. Ce qui est d'ailleurs très frappant et récurrent dans l'œuvre d'Henry Bauchau est le fait qu'il dépeint l'artiste en train de créer: ce dernier n'est plus seul dans son atelier et dans son espace. Toute la minutie, le doute, l'hésitation, la souffrance ou la violence de la création sont minutieusement décrits et observés. J'ai à l'esprit un passage tout à fait saisissant sur ce point, tiré de "L'arbre fou":

CEdipe est assis en face du tronc, il se lève souvent pour aller le toucher, sonder les nœuds, les torsions et les mouvements du bois, Parfois, une sorte de gémissement sort de sa bouche devant la difficulté de la tâche. Il soupèse la hache, la soulève, la soupèse, la repose sans se décider. Enfin, il s'élance, se rue vers le tronc. Avec de grands cris, il donne un coup, deux coups [... ${ }^{165}$

Dans L'Enfant bleu, le cheminement est identique. Il y a d'abord "rencontre " avec l'arbre en un corps à corps vibratoire particulier, puis passage à l'acte de création, d'abord par le trait, par le dessin. Je donnerai l'exemple du moment où, au cours d'une promenade, Orion et Véronique choisissent un arbre, un platane, pour l'enlacer afin de se charger de son énergie vitale:

Nous plaçons nos mains sur l'énorme tronc, je sens les ondes monter en moi [...] [Orion] les sent aussi. La matière le calme, parle à travers ses mains, il y a une présence tangible, sensible qui agit dans son corps et apaise son esprit. ${ }^{166}$

162 Henry Bauchau, Les Vallées du bonheur profond, Arles, Actes Sud, 1999, p. 9.

163 Ibid., p. 10.

164 Idem.

165 Idem.

166 Henry Bauchau, L'Enfant bleu, op. cit. p. 205. 
Véronique baptise l'arbre qu'a dessiné Orion après son expérience dans le parc, d'arbre

[...] intérieur, qu'il a découvert [...] là où il était, en lui-même. Dans son être meurtri, blessé, ligoté, il y a donc ce maître à demi enseveli, ce voyant de la vie aveugle. ${ }^{167}$

Quelque temps plus tard, Orion étend son expression artistique à la sculpture. Il sculpte sur bois une tête de bison dont Vasco évoque le pouvoir thérapeutique au toucher:

Il est en bois mais c'est aussi un vrai bison. Vous pouvez le caresser, cela vous fera du bien.

Leurs mains s'approchent, la douceur des courbes, la force vitale du bois les pénètrent et les rassurent. ${ }^{168}$

Ainsi, l'expression pleine de l'art se traduit par un impact réel, un pouvoir effectif sur le corps de celui qui touche ou perçoit. Mais ce n'est pas tout. Lacte de création dépeint dans L'Enfant bleu se veut aussi une expérience immédiate. Ceci est particulièrement frappant lorsqu'Orion décide de mettre le feu à ses dessins :

Il brûle certains dessins qu'il fait pour le feu, explique Véronique. Il pense qu'en brûlant ils parviennent à un instant d'intensité supérieure [...] C'est troublant pour nous qui attendons de l'art une certaine durée. La beauté instantanée, à demi perdue déjà, Orion la découvre dans le feu [...]. ${ }^{169}$

Il ne s'agit pas seulement d'une recherche d'intensité. C'est un acte de destruction qui anéantit du même coup les tensions ou problèmes évoqués par le dessin. L'amour impossible entre Orion et Myla, entravé par le père de celle-ci, s'annihile dans la consumation:

On voudrait, pour penser à Myla autrement, revoir l'enfant bleu et la petite fille sauvage. On voudrait brûler avec toi la lettre refusée où ils sont peints [...] on voudrait que le ménage, le mariage qu'on ne peut pas, on aimerait que tu les voies en feu avec moi. ${ }^{170}$

167 Ibid. p. 209. Une nouvelle fois apparaît ici la cécité comme manière de Voir. 
Cet acte s'apparente alors à un acte sacré ou magique, qui décuple la force du dessin et agit d'un point de vue symbolique et empirique. La destruction par le feu du noud émotionnel, c'est la destruction ipso facto de ce noud. C'est aussi l'abolition même, dans l'espace et le temps, de l'existence de ce moment douloureux.

Comment ne pas songer, dans le passage que nous venons d'évoquer et dans les pages consacrées à ce rituel dans L'Enfant bleu, à Antonin Artaud. De 1937 à 1944, il a envoyé à différents destinataires, des médecins, des amis ou certaines femmes de son entourage, des lettres-totems, des lettres où se mêlent écriture et dessin, qui, selon leur auteur, agissaient sur leurs destinataires tels des sorts protecteurs ou au contraire destructeurs: "S'y annonce la fonction d'exorcisme et d'insurrection [...], s'y définit la nécessité d'un acte d'ex-pression totale, où fonctionnent ensemble écriture et dessin, gestualité et oralité "171. De sa libération en 1946, à sa mort deux ans plus tard, les dessins d'Artaud verront se fondre dessins et écritures, parfois mais pas toujours glossolaliques. Avant cela encore, en 1937, dans Les Nouvelles Révélations de l'Être, Artaud donne la signification de ces brûlures: "brûler est une action magique et [...] il faut consentir à brûler, brûler d'avance et tout de suite, non pas une chose, mais tout ce qui pour nous représente les choses, pour ne pas s'exposer à brûler tout entiers "172. Ainsi bascule-t-on alors non dans la destruction, mais dans une forme de décréation weilienne. "Décréation: faire passer du créé dans l'incréé. Destruction: faire passer du créé dans le néant "173.

J'ai tâché au cours de ma réflexion de mettre en valeur certains aspects de la conception bauchalienne de l'art et de l'artiste/artisan. Je me suis aidée pour cela des ténus fils d'or qui lient Henry Bauchau, Simone Weil et Antonin Artaud, dans une certaine parenté spirituelle, métaphysique et intellectuelle. Tous trois ont ainsi évoqué dans leurs écrits, et dans leur œuvre plastique pour deux d'entre eux, une naissance à soi après le difficile combat de construction ou de destruction du "moi", la lutte contre les forces d'empêchement propres à la condition humaine, la dure nécessité, puis l'effacement de soi, l'effacement de ce "moi" dans l'œuvre, consenti ou refusé. Ils ont évoqué l'obéissance à une instance supérieure à l'imaginaire, au sens où l'entend Simone Weil et que nous avons développée dans ces pages, une obéissance à une nécessité d'où peut émerger, sinon la grâce, tout au moins l'espoir de la grâce. Mais alors que cette nécessité pousse Artaud dans une lutte lumineuse certes, mais souffrante, alors qu'elle consume Simone Weil à l'âge de 34 ans, elle pousse Henry Bauchau dans l'Acceptation au sens

171 Agnès de la Beaumelle, Antonin Artaud - Euvres sur papier, Paris, RMN, 1995, p. 85.

172 Antonin Artaud, Euvres complètes, vol. VII, Paris, Gallimard, 1982, p. 143. En italiques dans le texte.

173 Simone Weil, op. cit. p. 42. 
plein du terme, c'est-à-dire celle qui accueille les interrogations, contradictions, combats intérieurs qui font, qui fondent l'être humain.

"Natif de mes ruines surgissantes" ${ }^{174}$. Ce sont les mots choisis par Henry Bauchau pour accompagner son dessin intitulé "Larbre qui devient oiseau" et qui clôt L'Atelier spirituel. Pesanteur et grâce mêlées.

Être au monde. Être DANS le monde. Et aussi Être debout. Être ARBRE. Être tel Stéphane, l'ami du narrateur dans Le Boulevard périphérique: "Nous nous installons sous l'arbre, l'ombre y est tiède et fraîche [...]. Nous avons tous deux le dos appuyé à l'arbre. [Stéphane] n'éprouve pas la fraîcheur de l'ombre, il y est tout entier. Il est l'ombre comme tout à l'heure il sera le rocher" ${ }^{175}$.

La grâce. C'est donc possible?...

Irène Poutier

Besançon

174 Henry Bauchau, L'Atelier spirituel, Arles, Actes Sud, 2008, p. 78.

175 Henry Bauchau, Le Boulevard périphérique, op. cit., p. 56. 